\title{
Sufentanil sublingual tablet system versus oral oxycodone for management of postoperative pain in enhanced recovery after surgery pathway for total knee arthroplasty: a randomized controlled study
}

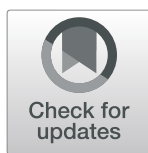

Emmanuel Noel ${ }^{1 *}$ (D) Luca Miglionico ${ }^{1}$, Mickael Leclercq ${ }^{1}$, Harold Jennart ${ }^{2}$, Jean-François Fils ${ }^{3}$ and Nicolas Van Rompaey

\begin{abstract}
Purpose: Effectiveness of sufentanil sublingual tablet system (SSTS) compared to oral oxycodone in the management of postoperative pain after total knee arthroplasty (TKA) within an enhanced recovery after surgery (ERAS) protocol.

Methods: This pragmatic, parallel, open label, randomized controlled, trial enrolled 72 adult patients scheduled for TKA under spinal anesthesia following ERAS pathway. In addition to multimodal analgesia, patients received SSTS 15 mcg (SSTS group) or oral oxycodone extended release $10 \mathrm{mg}$ twice daily and oral oxycodone immediate-release 5 $\mathrm{mg}$ up to four times daily on demand (Oxy group) to control pain during $48 \mathrm{~h}$ postoperatively. The primary endpoint was pain measured using a numeric rating scale at $24 \mathrm{~h}$ postoperatively. Time to first mobilization, side effects and patient satisfaction were also recorded.

Results: Median pain score at $24 \mathrm{~h}$ at rest was 3 [2-4] for Oxy group vs 2 [1.75-3] for SSTS group $(p=0.272)$ whereas median pain score on movement was $4[3-6]$ vs 3 [2-5] respectively $(p=0.059)$. No difference in time to first mobilization was found between the two groups. The method of pain control was judged good/excellent for 83.9\% of patients in the SSTS group compared with 52.9\% in the Oxy group $(p=0.007)$. The incidence of nausea was 33\% in SSTS group and 9\% in Oxy group ( $p=0.181)$.

Conclusions: In complement to ERAS multimodal analgesia, sublingual sufentanil 15 mcg tablet system did not show clinically significant pain improvement compared to oral oxycodone after total knee arthroplasty.

Trial registration: Clinical Trials: NCT04448457; retrospectively registered on June 24, 2020. https://clinicaltrials.gov/ ct2/show/NCT04448457? cond=sublingual +sufentanil\&cntry=BE\&draw=2\&rank=3
\end{abstract}

Keywords: Postoperative pain, Opioids, Multimodal analgesia, Fast track, Knee arthroplasty, Oxycodone, Sufentanil

\footnotetext{
*Correspondence: enoel@chu-tivoli.be

'Anesthesiology, CHU Tivoli Hospital, Avenue Max Buset, 34, 7100 La

Louvière, Belgium

Full list of author information is available at the end of the article
}

严 Springer Open (c) The Author(s). 2020 Open Access This article is licensed under a Creative Commons Attribution 4.0 International License, which permits use, sharing, adaptation, distribution and reproduction in any medium or format, as long as you give appropriate credit to the original author(s) and the source, provide a link to the Creative Commons licence, and indicate if changes were made. The images or other third party material in this article are included in the article's Creative Commons licence, unless indicated otherwise in a credit line to the material. If material is not included in the article's Creative Commons licence and your intended use is not permitted by statutory regulation or exceeds the permitted use, you will need to obtain permission directly from the copyright holder. To view a copy of this licence, visit http://creativecommons.org/licenses/by/4.0/. 


\section{Introduction}

Total knee arthroplasty (TKA) is a common procedure expected to grow in the near future [24]. To cope with this increasing demand, optimization of hospitalized patient pathway without compromising quality of care is required. Enhanced Recovery After Surgery (ERAS) protocols, through multidisciplinary approach and protocol-driven pathways, meet these goals in major orthopedic surgeries, reflected by a decrease in length of stay, complications and better patient satisfaction [7, 11, 25, 27]. One of the major components of ERAS is early mobilization which relies on minimally invasive surgical techniques, shortacting anesthetics, prevention of nausea and pain control through multimodal analgesia [3, 10, 14]. Despite increasing knowledge about postoperative pain management, the use of nonsteroidal anti-inflammatory drugs (NSAIDs), corticosteroids and local infiltration analgesia (LIA) techniques, moderate to severe pain is still experienced by many patients after TKA and opioids are still necessary [1]. Intravenous (IV) opioids patient-controlled analgesia (PCA) is the gold standard for acute postoperative pain management but the need of an indwelling catheter is considered as a limiting factor for early ambulation [14, 20]. In this context, oral drug administration is privileged, when used as a part in a multimodal regimen, oral oxycodone demonstrated better pain relief over unimodal intravenous opioid [13].

Sufentanil is a potent synthetic $\mu$ receptor-specific agonist which is mainly used for intraoperative surgical analgesia. When given via sublingual route sufentanil shows a rapid onset due to high lipophilicity, a good bioavailability (60\%) and a prolonged duration of action in comparison with IV sufentanil [4, 19]. Those characteristics make sublingual sufentanil a good candidate for postoperative pain treatment. The sufentanil sublingual tablet system (SSTS) (Zalviso ${ }^{\circ}$, Grünenthal $\mathrm{GmbH}$, Aachen, Germany) is a new, pre-programmed, noninvasive, handheld system device for patient-controlled analgesia [4, 16]. This system combines the advantages of non-IV route and self-administration, which could fit patients' needs in ERAS settings. The efficacy on postoperative pain relief has been shown in randomized controlled trials (RCT) for abdominal surgeries [16, 18, 21], plastic surgery [12] and major orthopedic surgeries [8, 16]. However, to our knowledge, use of SSTS in ERAS protocols has been reported only in few observational studies which conclude on the efficacy and the safety of the technique $[23,26,29]$. RCT comparing SSTS to an oral opioid effective regimen are also lacking.

Therefore, we conducted this trial to compare the efficacy of SSTS to oral oxycodone in the management of postoperative pain after TKA within an ERAS protocol. We hypothesized that SSTS could show a better profile on pain control, mobilization and patient satisfaction.
The primary endpoint of this study was to compare the effect of SSTS with oral oxycodone on pain intensity at $24 \mathrm{~h}$ postoperatively.

\section{Methods \\ Study design}

The study was a pragmatic, single-center, prospective, open label, randomized controlled trial conducted at CHU Tivoli Hospital, La Louvière, Belgium, to show the superiority of SSTS over oral oxycodone on pain control after total knee arthroplasty. The study design was approved by the Ethical Committee of Erasmus University Hospital, Brussels (ref P2017/348 on 21/06/2017). All patients signed an informed consent and the study was performed in accordance with the declaration of Helsinki. The study was registered retrospectively with ClinicalTrials.gov (NCT04448457). Inclusion criteria were adult patients (age $\geq 18$ years) scheduled for unilateral total knee arthroplasty under spinal anesthesia, American Society of Anesthesiologists (ASA) class 1-3. Exclusion criteria were contraindications to our ERAS protocol, allergy to study medications, history of addiction or preoperative chronic use of opioids, unicompartmental or revision knee replacement.

\section{Perioperative management}

All patients received oral midazolam, dosed at the discretion of the anesthesiologist, as anxiolytic premedication. Following the institutional ERAS protocol, $2 \mathrm{~g}$ cefazolin, $125 \mathrm{mg}$ methylprednisolone and $1 \mathrm{~g}$ of tranexamic acid were administered intravenously $30 \mathrm{~min}$ prior to incision. Spinal anesthesia was performed with 8-12 mg of hyperbaric bupivacaine $0.5 \%$ without adjuvant. As part of the multimodal pain management protocol, all patients received by the surgeon local infiltration analgesia with $200 \mathrm{~mL}$ of ropivacaine $0,2 \%$ (with adjunction of epinephrine $2,5 \mathrm{mcg} / \mathrm{mL}$ ). At the end of the surgery 3 $\mathrm{g}$ of tranexamic acid diluted in $70 \mathrm{~mL}$ of $\mathrm{NaCl} 0.9 \%$ were injected intraarticularly. All surgical procedures were performed by the same three surgeons without tourniquet and no drain was left in place.

At the arrival in post-anesthesia care unit (PACU), if necessary, pain was initially treated with IV piritramide until discharge criteria to the ward were met. For the postoperative multimodal pain management, application of ice pack on the wound area, acetaminophen $1 \mathrm{~g}$ PO four times daily and celecoxib $200 \mathrm{mg}$ PO one time daily were given systematically to all patients. Postoperative nausea and vomiting (PONV) prophylaxis was administered according to institutional guidelines.

Patients followed our institutional rehabilitation protocol which consists of lifting the patient by the physiotherapist as soon as he returns from the recovery room, the patient is also seated whenever it's possible on day 0 
(day of surgery). On day 1, the patient starts passive motion machine (Kinetec ${ }^{\circ}$, Tournes, France), exercises in the physiotherapy room, occupational therapy program, and so on, on day 2.

\section{Randomization and intervention}

Patients were randomly assigned to study groups (allocation ratio 1:1) in fixed blocks of 4 using computergenerated random numbers kept in sealed envelopes. Envelope was opened at the arrival of the patient in the operating theater and subjects were allocated to the sublingual sufentanil tablet system (SSTS) group or the oral oxycodone (Oxy) group.

Patients enrolled in the SSTS group were instructed for the use of the device in PACU and the first dose was administered there allowing sublingual administration of sufentanil $15 \mathrm{mcg}$ tablet with a fixed 20-min lockout interval. In the Oxy group, patients received oral oxycodone extended-release (ER) $10 \mathrm{mg}$ twice daily and oral oxycodone immediate-release (IR) $5 \mathrm{mg}$ up to four times daily on demand when numeric rating scale $(\mathrm{NRS})>3$. In case of insufficient pain management, patients could receive rescue intramuscular morphine injection and patients would be automatically excluded from the study.

\section{Outcomes assessment}

Primary endpoint was pain score at $24 \mathrm{~h}$ postoperatively. Pain was assessed using a verbal 11-point numeric rating scale (NRS-11) ranging from 0 ("No pain") to 10 ("Worst imaginable pain"). The verbal NRS-11 is a well validated, reliable and sensitive tool to evaluate acute postoperative pain $[2,6]$. Patients were asked to evaluate their pain at rest (PAR) and pain evoked by passive or active flexion of the knee (movement evoked pain - MEP). Secondary outcomes were pain scores measured $2 \mathrm{~h}, 6 \mathrm{~h}$ after surgery and every $6 \mathrm{~h}$ thereafter up to $48 \mathrm{~h}$, success of first mobilization (with or without aid) assessed by the first upright standing position followed by success of mobilization at these different timepoints, nausea and vomiting and finally satisfaction for the method of pain control assessed using Patient Global Assessment (PGA) of the method of pain control questionnaire at the completion of the $48 \mathrm{~h}$ study period which consist on a 4point categorical scale, where $1=$ poor, $2=$ fair, $3=\operatorname{good}$ and $4=$ excellent [22].

\section{Statistical analysis}

Sample size calculation was estimated on the basis of an absolute reduction of NRS score in SSTS group compared to Oxy group. Analysis of pain scores obtained before the start of the study on 7 patients treated by oxycodone indicated a mean pain score of 5.587 with a standard deviation of 1.46 at $24 \mathrm{~h}$ after surgery. In order to show a difference of 2 points of NRS score with a power of 0.9 and a bilateral alpha risk of 0.05 we calculate the need of 28 patients per group, considering a minimal clinically important difference of 1 . Taking into account an estimated drop-off of $20 \%$, we conclude that a total of 70 patients were needed. We used the 'Trial Size' package of the $\mathrm{R}$ software to perform the sample size calculation.

Continuous data were compared by means of T-test when homogeneity of variances, tested with the Bartlett's test, and normality of the residuals, tested with the Shapiro-Wilks test, were reached and means and standard deviations (SD) are reported. When homogeneity of the variance or normality of the residuals were not proved, Wilcoxon signed rank test was performed on rank data and medians and interquartile ranges $\left(\left[\mathrm{Q}_{25}-\right.\right.$ $\left.\mathrm{Q}_{75}\right]$ ) are reported. For count data, the Pearson ChiSquared test was performed to compare proportions. For time to event data, Kaplan-Meier product-limit estimators of cumulative rates of patients reaching the event (success of first mobilization) at follow-up time points were calculated. A log-rank test was used to compare the two treatment groups. We used the software R, version 3.4.3 (R Core Team, 2017) to perform the statistical analyses. Prism version 8.4.2 (GraphPad Software, Inc.) was used to construct the figures.

\section{Results}

One hundred-seven patients were screened for eligibility between September 2017 and July 2018. Seventy-two patients were recruited and prospectively randomized between the two groups, 69 patients completed the followup at $24 \mathrm{~h}$ and 66 patients at $48 \mathrm{~h}$. Three patients stopped prematurely the study due to nausea discomfort, one was excluded due to pain control insufficiency, one asked to leave the hospital at day 1 and one patient died in the afternoon at day 0 (Fig. 1). Because no relationship was found between the use of medications and the death, the Ethical Committee gave his approval to continue the study. There were no differences in the baseline characteristics between the two groups (Table 1). Median piritramide consumption in the PACU was 0 [0-2.75] for Oxy group vs 0 [0-0] for SSTS group $(p=$ 0.239). During the $48 \mathrm{~h}$ observation period, all patients in the Oxy group received $50 \mathrm{mg}$ of oxycodone ER, the mean oxycodone IR consumption was 33.6 (7.8) $\mathrm{mg}$. In the SSTS group, the mean tablet consumption was 14.4 (7.84) tablets or 215.4 (117.6) mcg of sufentanil. Concerning the primary endpoint, pain score at $24 \mathrm{~h}$, there was no statistical difference found between the two groups. Median pain score at rest was 3 [2-4] Oxy group vs 2 [1.75-3] for SSTS group ( $p=0.272)$ whereas median dynamic pain score was 4 [3-6] vs 3 [2-5] without hyperlink respectively $(p=0.059) \quad$ (Fig. 2). Nine patients $(25.7 \%)$ described moderate-to-severe pain (NRS $\geq 4)$ in 


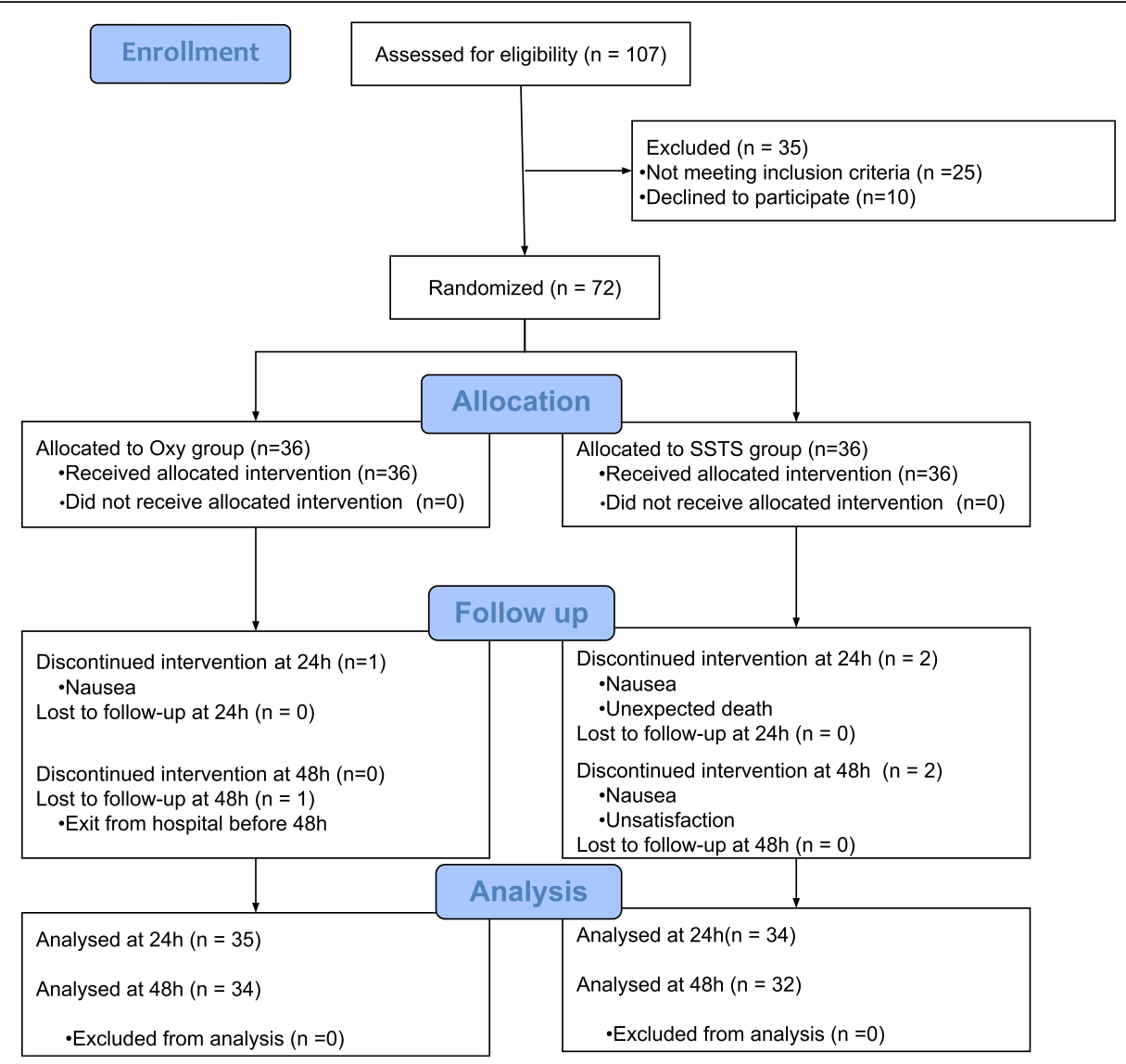

Fig. 1 Participant Flow (CONSORT diagram). SSTS = sufentanil sublingual tablet system

Oxy Group vs five patients $(14.7 \%)(p=0.255)$ in SSTS group at rest, and $23(65.7 \%)$ vs $15(44.1 \%)(p=0.071)$ respectively on movement. No statistical differences were found neither in static nor in dynamic pain over the $48 \mathrm{~h}$ postoperative period (Fig. 3). Seven patients (9\%) complained of nausea in the Oxy group and 12 patients (33\%) in the SSTS group (RR 0.58, 95\% confidence interval $[0.260$ to 1.270$], p=0.181)$. Three $(8 \%)$ patients suffered vomiting in the Oxy group and $4(11 \%)$ in the SSTS group $(p=0.690)$. One patient complained of pruritus in the Oxy group. As shown in Fig. 4, time to first mobilization was not different between groups. At the end of the study, the assessment of the method of pain control was judged Good/Excellent for $26 / 31$ (83.9\%) patients in the SSTS group compared with $18 / 34$ (52.9\%) in the Oxy group ( $p=0.007)$ (Fig. 5).

\section{Discussion}

In our pragmatic prospective randomized study, SSTS 15 mcg showed no clinical benefit compared to a conventional oxycodone-based treatment for postoperative

Table 1 Patients' characteristics

\begin{tabular}{llll}
\hline & $\begin{array}{l}\text { Oxy group } \\
(\boldsymbol{n}=\mathbf{3 5 )}\end{array}$ & $\begin{array}{l}\text { SSTS group } \\
(\boldsymbol{n}=\mathbf{3 4 )}\end{array}$ & $\begin{array}{c}\boldsymbol{p} \text {-value } \\
\text { Age (years) }\end{array}$ \\
Sex (woman) & $65.3 \pm 9.0$ & $67.6 \pm 8.2$ & 0.262 \\
ASA status (1/2/3) & $20(57.1 \%)$ & $21(61.7 \%)$ & 0.807 \\
Weight (kg) & $9 / 22 / 4$ & $9 / 23 / 2$ & - \\
Height (cm) & $90[78-100]$ & $87.5[79.75-98]$ & 0.874 \\
BMl $\left(\mathrm{kg} / \mathrm{m}^{2}\right)$ & $166.6 \pm 9.3$ & $166.4 \pm 9.6$ & 0.868 \\
\hline
\end{tabular}

Values are expressed as mean \pm standard deviation, median with interquartile range [Q25-Q75] or number and percentage $B M I$ Body Mass Index, SSTS sufentanil sublingual tablet system 

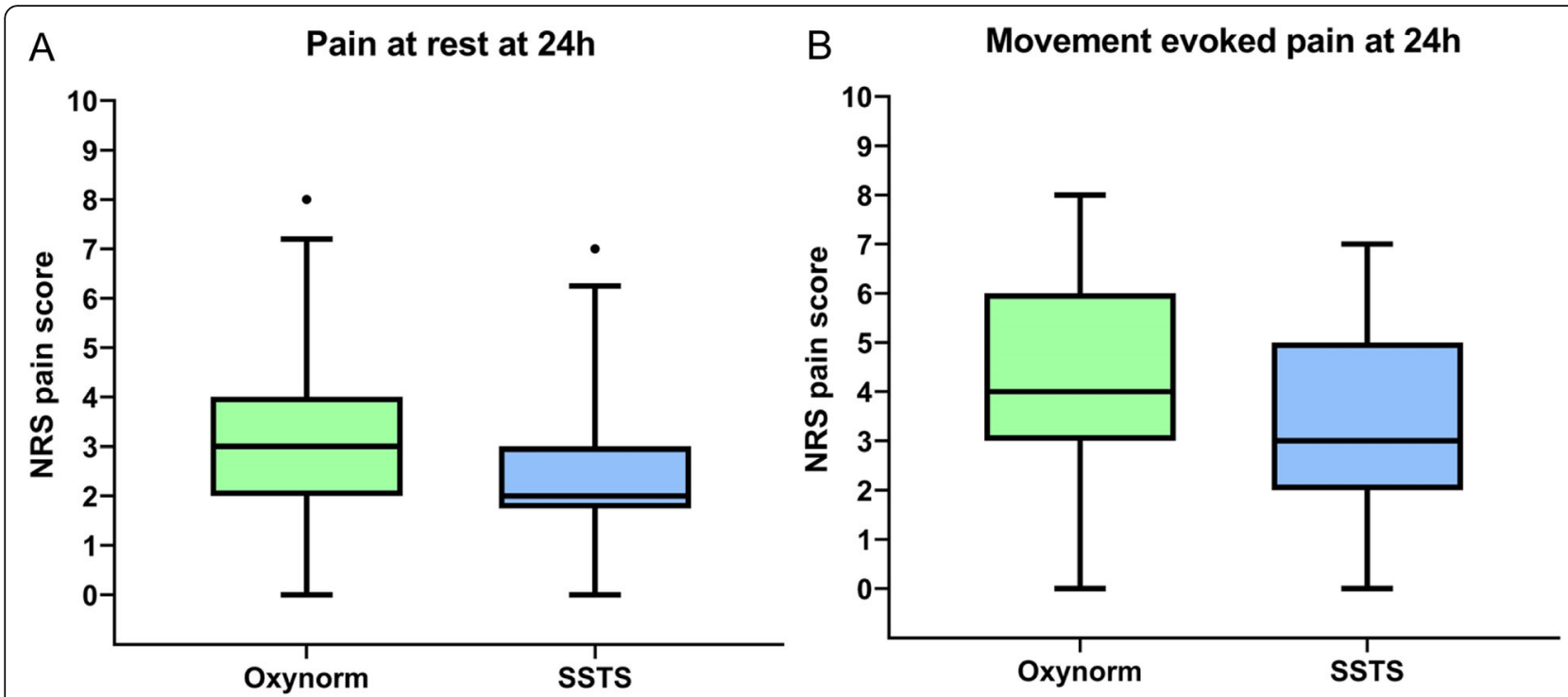

Fig. 2 Box and whisker plots of postoperative pain score at $24 \mathrm{~h}$ a. Pain score at rest. b. Dynamic pain score. Solid horizontal lines represent the median, boxes indicate interquartile range (Q25-Q75), whiskers represent 5th percentile and 95th percentile and dots are the extreme values. No statistical difference was found

pain management in a population of TKA patients managed with our ERAS protocol. The lack of meaningful effect on pain score was noted for pain at rest but also for movement-evoked pain, a critically important outcome measure especially in the context of TKA [5]. Our data underlines the fact that, even with multimodal analgesia, TKA remains a painful procedure as seen in other studies [9]. While SSTS $15 \mathrm{mcg}$ is indicated for the management of moderate to severe acute postoperative pain, SSTS treated patients showed no significant pain improvement and almost $50 \%$ of the patients are still experiencing NRS $\geq 4$ on movement. Moreover, only one patient needed rescue analgesia and he was treated in the SSTS group.

Comparison to other studies is limited because few RCT addressed specifically the role of SSTS on postoperative pain. Compared to placebo without any coanalgesics, SSTS $15 \mathrm{mcg}$ and $20 \mathrm{~min}$ lockout interval improved mean [SEM] summed pain intensity difference (SPID) from baseline over $48 \mathrm{~h}$ (SSTS 105.6 [10.14] vs placebo 55.58 [13.11]) after open abdominal surgeries [21]. Similar effects were observed in orthopedic surgeries where Jove et al. [8] showed that STSS $15 \mathrm{mcg}$ could improve SPID $48 \mathrm{~h}$ after TKA or total hip arthroplasty (SSTS 76 [7] vs placebo -11 [11]). In this study, mean NRS pain scores at $24 \mathrm{~h}$ were $3.9( \pm 0.2)$ for SSTS versus $5.1( \pm 0.4)$ for placebo $(p=0.002)$, one could argue on the clinical relevance of this result. The lack of multimodal analgesia as well as the absence of an effective control group could explain the pronounced effect of SSTS in these studies. This hypothesis is supported by the lack of difference on postoperative pain scores when SSTS was compared with continuous morphine after cardiac surgery [28]. After major orthopedic surgeries, no differences between SPID over 24, 48 or $72 \mathrm{~h}$ was seen when compared to intravenous morphine PCA for the management of acute postoperative pain although faster analgesia was observed in the first $4 \mathrm{~h} \mathrm{[16].} \mathrm{Be-}$ cause IV PCA restricts mobility, we decided to use as active comparator oral oxycodone treatment. SSTS and oral oxycodone have approximately the same onset $(15-30 \mathrm{~min})[18,21]$ but SSTS does not expose patients to prolonged analgesic gaps due to absence of involvement of nurses in analgesic administration. Lack of gap and better titration should optimize pain control as seen with other PCA systems as suggested previously [23]. This optimized analgesia was not reflected in our study neither in a retrospective trial on 227 patients operated for TKA in an ERAS environment [29]. Similarly, to our study, all patients received ropivacaine LIA, acetaminophen and NSAIDS (metamizole three times daily) for multimodal analgesia. SSTS $15 \mathrm{mcg}$ treated patients $(n=72)$ were compared to those who received oral oxycodone $(n=$ 68 ) or oral oxycodone with dexamethasone given preoperatively $(n=87)$. Lowest and highest NRS pain scores at rest were compared during the first postoperative $48 \mathrm{~h}$ but SSTS showed no improvement on pain scores. Of note, sufentanil consumption was in the same range observed in our study (mean 13.75 (11.96) tablets over $48 \mathrm{~h}$ ). 

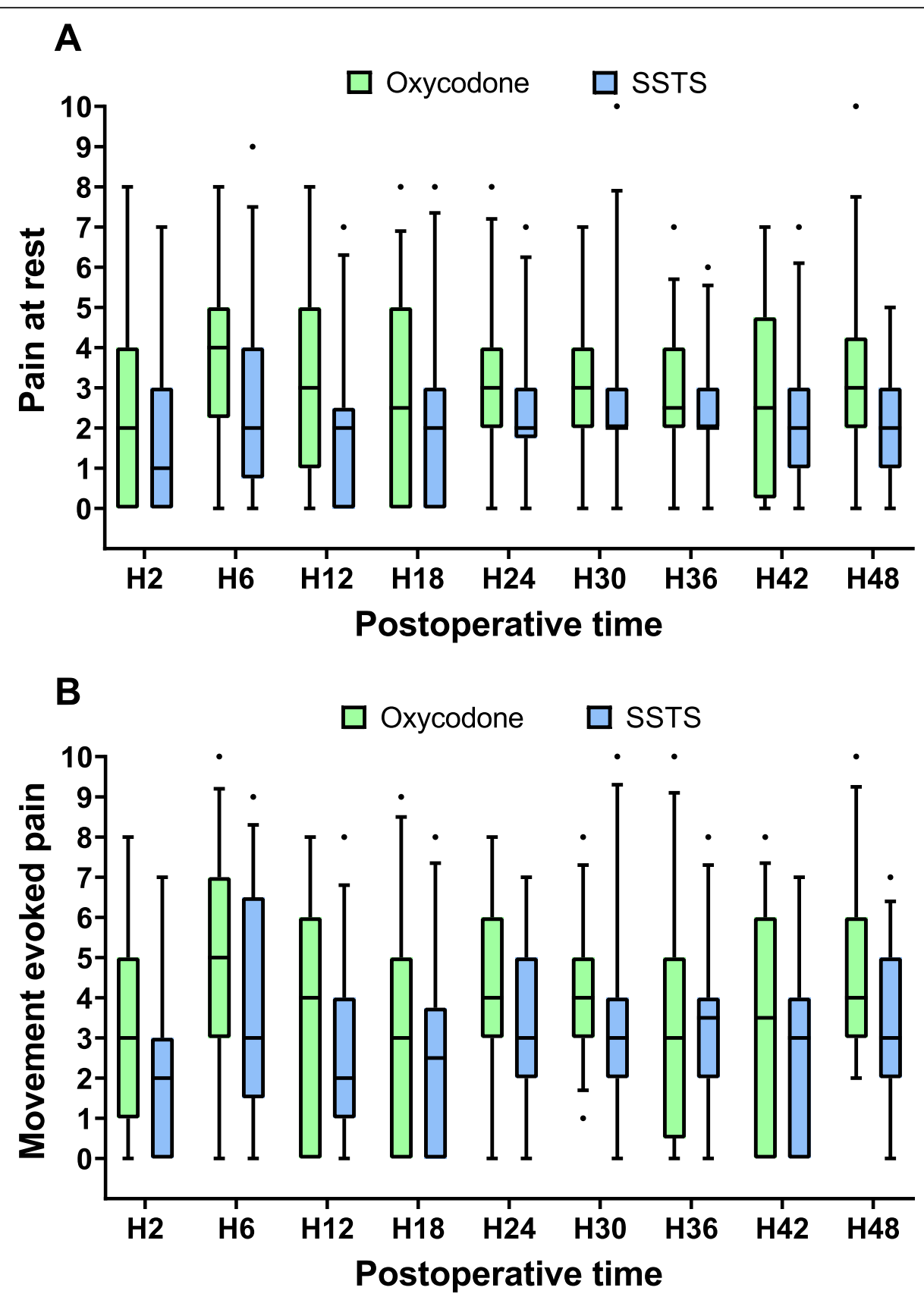

Fig. 3 Box and whisker plots of postoperative pain scores over $48 \mathrm{~h}$. a. Pain score at rest. b. Dynamic pain score. Solid horizontal lines represent the median, boxes indicate interquartile range (Q25-Q75), whiskers represent 5th percentile and 95th percentile and dots are the extreme values. No statistical difference was found. SSTS = sufentanil sublingual tablet system

In our ERAS pathway, more than $80 \%$ patients in both groups were mobilized on day 0 . With this high success of first mobilization the role of SSTS in functional outcome as early mobilization seems to be limited.

Incidence of adverse events was limited, but $33 \%$ of patients for SSTS group versus 9\% of patients for Oxy group reported nausea during the $48 \mathrm{~h}$ which is in accordance with the literature $[4,15]$. Even if this difference was not significant, which could be explained by the small sample size, nausea was the main reason for dropping out the study and should be taken into consideration. Although studies showed that SSTS could save time at the ward $[17,26,29]$, the most interesting advantage from the use of SSTS based on our study was the patient's satisfaction rate, as mentioned in other studies $[5,16]$. This satisfaction could be explained by the easy use of this new PCA device and the feeling of personalization due to the thumb tag, but a Hawthorne 


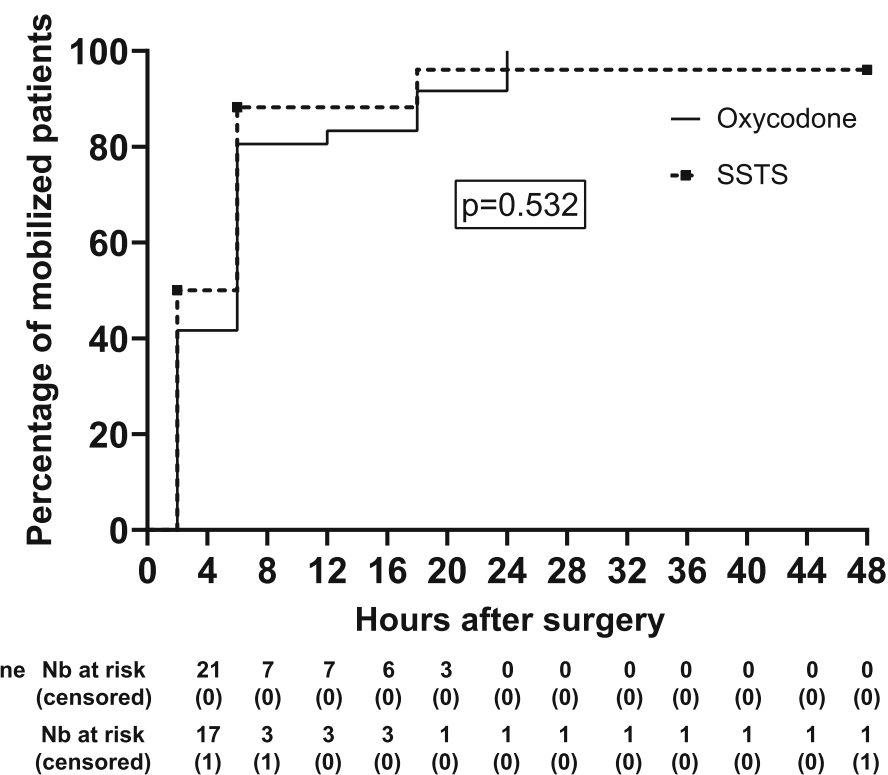

Fig. 4 Kaplan-Meier cumulative event rates for time to first mobilization. Solid square indicates censored patient. No statistical difference was found. SSTS = sufentanil sublingual tablet system

effect is probably involved because of the open blind design and the novelty of the device.

Concerning the use of the system, we found some issues with the operation of the device, we noticed always the same problem: the thumb tag had to be replaced for three patients, especially at the beginning of the study.

Regarding the limited efficacy of the system and the high prevalence of nausea, the economic burden seems not to be justified even if patient satisfaction is improved. Indeed, in our hospital, the cost of one tablet of Oxynorm IR is $€ 0.085$, Oxynorm ER is $€ 0.125$ and the cost of the 40 tablets cartridge is $€ 105$. Regarding the mean consumption in each group, the total cost over $48 \mathrm{~h}$ is $€ 1.195$ for Oxy group and $€ 105$ for SSTS group. The price of the device (around $€ 1400$ ) as well the cost of the thumb

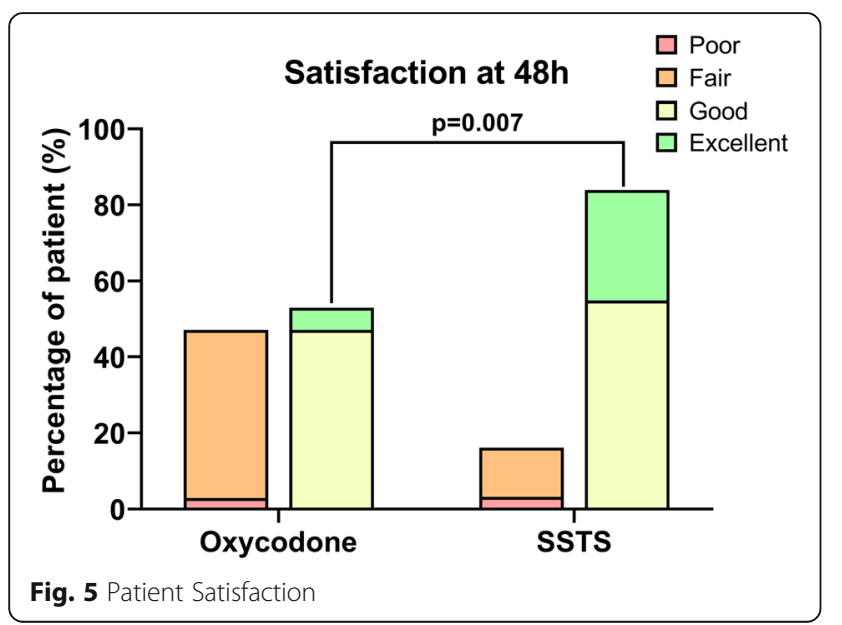

patch should also be taken into account. Our study had several limitations. The use of mean NRS at $24 \mathrm{~h}$ as the primary endpoint may be less sensitive than SPID to detect a difference between treatment. The open label design could have influenced the PGA as explained above.

\section{Conclusion}

In complement to ERAS multimodal analgesia, sublingual sufentanil $15 \mathrm{mcg}$ tablet system did not show significant pain improvement compared to oral oxycodone after total knee arthroplasty.

\section{Acknowledgements}

We would like to thank the pain management nurses for their assistance with this study.

\section{Authors' contributions}

E N: helped design the study, conduct the study, analyze the data, write the manuscript. $L \mathrm{M}$ : helped design the study, conduct the study, collect the data, prepare the manuscript. M L: helped design the study, conduct the study, analyze the data and prepare the manuscript. $\mathrm{H} \mathrm{J}$ : helped in patient recruitment and reviewed the manuscript. JF F: helped in statistical analysis. $\mathrm{N}$ VR: helped analyze the data and write the manuscript. All authors read and gave final approval of the version to be published. The author(s) read and approved the final manuscript.

\section{Availability of data and materials}

The datasets used and/or analyzed during the current study are available from the corresponding author on reasonable request.

Ethics approval and consent to participate

All patients signed an informed consent and the study was performed in accordance with the declaration of Helsinki. The study design was approved by the Ethical Committee of Erasmus University Hospital, Brussels (ref P2017/ 348 on 21/06/2017). 


\section{Consent for publication}

Not applicable.

\section{Competing interests}

The authors have no disclosure for this research.

Grünenthal GmbH provided our hospital with non-financial support. Specifically, this company offered a free rental of the zalviso administration device (sufentanil cartridge not included) without any compensation.

\section{Author details}

'Anesthesiology, CHU Tivoli Hospital, Avenue Max Buset, 34, 7100 La Louvière, Belgium. ${ }^{2}$ Orthopedic surgery, CHU Tivoli Hospital, La Louvière, Belgium. ${ }^{3}$ ARS Statistica, statistics, Nivelles, Belgium.

Received: 4 September 2020 Accepted: 2 November 2020

Published online: 20 November 2020

\section{References}

1. Albrecht E, Guyen O, Jacot-Guillarmod A, Kirkham KR (2016) The analgesic efficacy of local infiltration analgesia vs femoral nerve block after total knee arthroplasty: a systematic review and meta-analysis. Br J Anaesth 1;116(5): 597-609. doi: https://doi.org/10.1093/bja/aew099

2. Breivik EK, Björnsson GA, Skovlund E (2000) A comparison of pain rating scales by sampling from clinical trial data. Clin J Pain 16(1):22-28. https:// doi.org/10.1097/00002508-200003000-00005

3. den Hertog A, Gliesche K, Timm J, Mühlbauer B, Zebrowski S (2012) Pathway-controlled fast-track rehabilitation after total knee arthroplasty: a randomized prospective clinical study evaluating the recovery pattern, drug consumption, and length of stay. Arch Orthop Trauma Surg 132(8):11531163. https://doi.org/10.1007/s00402-012-1528-1

4. van de Donk T, Ward S, Langford R, Dahan A (2018) Pharmacokinetics and pharmacodynamics of sublingual sufentanil for postoperative pain management. Anaesthesia 73(2):231-237. https://doi.org/10.1111/ anae.14132

5. Gilron I, Kehlet H, Pogatzki-Zahn E (2019) Current status and future directions of pain-related outcome measures for post-surgical pain trials. Can J Pain 15;3(2):36-43. doi: https://doi.org/10.1080/24740527.2019. 1583044

6. Hjermstad MJ, Fayers PM, Haugen DF, Caraceni A, Hanks GW, Loge JH, Fainsinger R, Aass N, Kaasa S (2011) European palliative care research collaborative (EPCRC). Studies comparing numerical rating scales, verbal rating scales, and visual analogue scales for assessment of pain intensity in adults: a systematic literature review. J Pain Symptom Manag 41(6):10731093. 21621130. https://doi.org/10.1016/j.jpainsymman.2010.08.016

7. Jones EL, Wainwright TW, Foster JD, Smith JRA, Middleton RG, Francis NK (2014) A systematic review of patient reported outcomes and patient experience in enhanced recovery after orthopaedic surgery. Ann R Coll Surg Engl 96(2):89-94. https://doi.org/10.1308/003588414X13824511649571

8. Jove M, Griffin D, Minkowitz H, Ben-David B, Evashenk M, Palmer P (2015) Sufentanil sublingual tablet system for the Management of Postoperative Pain after knee or hip Arthroplasty. Anesthesiology 123(2):434-443. https:// doi.org/10.1097/ALN.0000000000000746

9. Karlsen AP, Wetterslev M, Hansen SE, Hansen MS, Mathiesen O, Dahl JB (2017) Postoperative pain treatment after total knee arthroplasty: a systematic review. PLoS One. 8;12(3): e0173107. doi: https://doi.org/10.1371/ journal.pone.0173107. eCollection 2017

10. Kehlet H, Wilmore DW (2008) Evidence-based surgical care and the evolution of fast-track surgery. Ann Surg 248(2):189-198. https://doi.org/10. 1097/SLA.0b013e31817f2c1a

11. Kehlet H, Thienpont E (2013) Fast-track knee arthroplasty -- status and future challenges. Knee 20(Suppl 1):S29-S33. https://doi.org/10.1016/S09680160(13)70006-1

12. Lakshman S, Minkowitz H, Melson T, Leiman D (2016) Abstract: safety and efficacy of Sufentanil sublingual 30 mcg tablets for the treatment of acute pain following outpatient Abdominoplasty. Plast Reconstr Surg Glob Open 4(9 Suppl):58-59. https://doi.org/10.1097/01.GOX.0000502942.62522.e4

13. Lamplot JD, Wagner ER, Manning DW (2014) Multimodal pain Management in Total Knee Arthroplasty. J Arthroplast 29(2):329-334. https://doi.org/10. 1016/j.arth.2013.06.005

14. Ljungqvist $O$ (2019) Enhanced recovery after surgery-knowing, not guessing. JAMA Surg.1; 154(8): 736-737.doi: https://doi.org/10.1001/jamasurg.2019.1008
15. Meijer F, Cornelissen P, Sie C, Wagemans M, Mars A, Hobma T et al (2018) Sublingual sufentanil for postoperative pain relief: first clinical experiences. Pain Res 11:987-992. https://doi.org/10.2147/JPR.S160091

16. Melson TI, Boyer DL, Minkowitz HS, Turan A, Chiang Y-K, Evashenk MA, Palmer P (2014) Sufentanil sublingual tablet system vs. intravenous patientcontrolled analgesia with morphine for postoperative pain control: a randomized, Active-Comparator. Trial Pain Pract 14(8):679-688. https://doi. org/10.1111/papr.12238

17. Meuser T, Nardi-Hiebl S, Eberhart L, Paul M, Böttger R, Reutershan J (2019) Staff time requirements for postoperative pain management: comparison of sufentanil sublingual tablet system and intravenous patient-controlled analgesia. J Opioid Manag 16(1):33-39. https://doi.org/10.5055/jom.2020.0548

18. Minkowitz HS, Leiman D, Melson T, Singla N, DiDonato KP, Palmer PP (2017) Sufentanil sublingual tablet 30 mcg for the Management of Pain Following Abdominal Surgery: a randomized, placebo-controlled, Phase-3 study. Pain Pract 17(7):848-858. https://doi.org/10.1111/papr.12531

19. Minkowitz HS, Singla N, Evashenk MA, Hwang SS, Chiang YK, Hamel LG, Palmer PP (2013) Pharmacokinetics of sublingual sufentanil tablets and efficacy and safety in the management of postoperative pain. Reg Anesth Pain Med 38(2):131-139. https://doi.org/10.1097/AAP.0b013e3182791157

20. Morlion B, Schäfer M, Betteridge N, Kalso E (2018) Non-invasive patientcontrolled analgesia in the management of acute postoperative pain in the hospital setting. Curr Med Res Opin 34(7):1179-1186. https://doi.org/10. 1080/03007995.2018.1462785

21. Ringold FG, Minkowitz HS, Gan TJ, Aqua KA, Chiang Y, Evashenk MA, Palmer P (2015) Sufentanil sublingual tablet system for the management of postoperative pain following open abdominal surgery. Reg Anesth Pain Med 40(1):22-30. https://doi.org/10.1097/AAP.0000000000000152

22. Rothman M, Vallow S, Damaraju CV, Hewitt DJ (2009) Using the patient global assessment of the method of pain control to assess new analgesic modalities in clinical trials. Curr Med Res Opin 25(6):1433-1443. https://doi. org/10.1185/03007990902862032

23. Scardino M, D'Amato T, Martorelli F, Fenocchio G, Simili V, Di Matteo B, Bugada D, Kon E (2018) Sublingual sufentanil tablet system Zalviso ${ }^{\circledR}$ for postoperative analgesia after knee replacement in fast track surgery: a pilot observational study. J Exp Orthop 20. https://doi.org/10.1186/s40634-018-0123-y

24. Sloan M, Premkumar A, Sheth NP (2018) Projected volume of primary Total joint Arthroplasty in the U.S., 2014 to 2030. JBJS 5:100(17):1455-1460. doi: https://doi.org/10.2106/JBJS.17.01617

25. Soffin EM, YaDeau JT (2016) Enhanced recovery after surgery for primary hip and knee arthroplasty: a review of the evidence. Br J Anaesth 117:II62-II172. https://doi.org/10.1093/bja/aew362

26. Turi S, Deni F, Lombardi G, Marmiere M, Nisi FG, Beretta L (2019) Sufentanil sublingual tablet system (SSTS) for the management of postoperative pain after major abdominal and gynecological surgery within an ERAS protocol: an observational study. J Pain Res 12:2313-2319. https://doi.org/10.2147/JPR.S214600

27. Turnbull ZA, Sastow D, Giambrone GP, Tedore T (2017) Anesthesia for the patient undergoing total knee replacement: current status and future prospects. Local Reg Anesth 10:1-7. https://doi.org/10.2147/LRA.S101373

28. Van Tittelboom V, Poelaert R, Malbrain MLNG, La Meir M, Staessens K, Poelaert J.J (2020) Sublingual Sufentanil Tablet System Versus Continuous Morphine Infusion for Postoperative Analgesia in Cardiac Surgery Patients. Cardiothorac Vasc Anesth. 19:S1053-S0770(20)30824-7. doi: https:/doi.org/ 10.1053/j.jvca.2020.08.030

29. van Veen DE, Verhelst CC, van Dellen RT, Koopman J (2018) Sublingual sufentanil (Zalviso) patient-controlled analgesia after total knee arthroplasty: a retrospective comparison with oxycodone with or without dexamethasone. J Pain Res 11:3205-3210. https://doi.org/10.2147/JPR.S185197

\section{Publisher's Note}

Springer Nature remains neutral with regard to jurisdictional claims in published maps and institutional affiliations. 